\title{
Multi-mode transport through a quantum nanowire with two embedded dots
}

\author{
Vidar Gudmundsson, ${ }^{1}$ Gudny Gudmundsdottir, ${ }^{1}$ Jens Hjorleifur Bardarson, ${ }^{1}$ \\ Ingibjorg Magnusdottir, ${ }^{1}$ Chi-Shung Tang, ${ }^{2}$ and Andrei Manolescu ${ }^{1}$ \\ ${ }^{1}$ Science Institute, University of Iceland, Dunhaga 3, IS-107 Reykjavik, Iceland \\ ${ }^{2}$ Physics Division, National Center for Theoretical Sciences, P.O. Box 2-131, Hsinchu 30013, Taiwan
}

\begin{abstract}
We investigate the conductance of a quantum wire with two embedded quantum dots using a $\mathrm{T}$ matrix approach based on the Lippmann-Schwinger formalism. The quantum dots are represented by a quantum well with Gaussian shape and the wire is two-dimensional with parabolic confinement in the transverse direction. In a broad wire the transport can assume a strong nonadiabatic character and the conductance manifests effects caused by intertwined inter- and intra-dot processes that are identified by analysis of the "nearfield" probability distribution of the transported electrons.
\end{abstract}

\section{INTRODUCTION}

Transport through vertical double dot systems has been investigated by many groups experimentally 1, 2, 3, 4, 5] or theoretically [6, 7, 8, 9, 10, 11, 12], just to mention few. In most of these models the researchers use effective Hubbard-type models or exact diagonalization for few electrons in order to describe the effects of higher order correlations on the electron transport. Other studies have focused on describing the effects of the interaction of the geometry of the leads and the quantum dot, where commonly, the system tracted is a dot embedded in a quantum wire [13, 14]. The focus has then been on resonances caused by the interplay of the discrete quasibound states of the dot and the continuum of the wire [15, 16].

Here we report our investigation on a system composed of a quasi two-dimensional quantum wire in no external magnetic field with a parabolic confinement in the direction transverse to the transport. Embedded in the quantum wire we have two identical quantum dots whose centers are separated by the distance $2 d$, but the effective size of the smoothly Gaussian shaped dots is on the order of $d / 8$. We shall consider a narrow wire chosen such that the depth of the dot is a bit larger than the energy separation of the unperturbed subbands of the wire, and a broad wire where the subband separation is only $1 / 10$ of the dot depth. We consider two different dot sizes, but in both cases the effective size of the dot does not broaden the wire. We are thus considering a wire with embedded dots in between the limit where the dots are similar to an impurity represented by attractive $\delta$-functions, and the case where the dots can be considered of the same width or wider than the wire. We assume the electrons to be incident in some of the lowest lying energy subbands of the wire, but due to the geometry of the system we have to include several more subbands in the calculation in order to describe the transport correctly.

\section{MODELS}

We consider a multi-mode transport of electrons along the $z$-direction through a two-dimensional quantum wire defined by a parabolic confinement in the $x$-direction with the characteristic frequency $E_{0}=\hbar \Omega_{0}$. The electrons incident from the left $(z \rightarrow-\infty)$ impinge on a scattering potential composed of two Gaussian wells located at $z= \pm d$

$$
V_{s c}=V_{0}\left\{e^{-\beta\left((z-d)^{2}+x^{2}\right)}+e^{-\beta\left((z+d)^{2}+x^{2}\right)}\right\} .
$$

The scattering modes are expanded in the transverse modes $\chi_{m}$

$$
\psi_{n E}^{+}(\mathbf{r})=\sum_{m} \varphi_{m E}^{n}(z) \chi_{m}(x)
$$

that are the eigenmodes of the parabolic confinement of the quantum wire. An incident electron with energy $E=\hbar^{2} k_{n}^{2}(E) /(2 m)+\epsilon_{n}$, where $\epsilon_{n}=\hbar \Omega_{0}(n+1 / 2)$ can propagate in mode $n$ if $\epsilon_{n}<E$ otherwise the state is evanescent. We use the coupled multi-mode LippmannSchwinger equations [17, 18] to establish a set of coupled integral equations for the $T$-matrix [19] which in turn is used to calculate the transmission amplitude that within the Landauer formalism leads to the linear response conductance at vanishing temperature [20, 21]

$$
G=\frac{2 e^{2}}{h} \operatorname{Tr}\left[t^{\dagger} t\right]
$$

The $T$-matrix can also be used to construct the probability density of the scattering states through their wave functions [19]

$$
\phi_{m E}^{n}(z)=\phi_{m E}^{n 0}(z)+\frac{m}{\pi \hbar^{2}} \int_{-\infty}^{+\infty} d p \frac{\sqrt{|p|} e^{i p z}}{k_{m}^{2}-p^{2}} T_{m n}\left(p, k_{n}\right) .
$$

All matrix elements have been calculated analytically and special care has been taken to include enough modes in the numerical calculations to reach convergent solutions. The singular part of the integration for the scattering states (4) and the $T$-matrix has been completed analytically [19].

The mirror symmetry of the scattering potential (1) along the center, $x=0$, of the wire results in a finite matrix elements only between transverse modes of the same parity. 


\section{RESULTS}

We shall consider a broad $\left(E_{0}=\hbar \Omega_{0}=1 \mathrm{meV}\right)$ or a narrow $\left(E_{0}=\hbar \Omega_{0}=6 \mathrm{meV}\right)$ quantum wire with two identical embedded quantum dots located at $z= \pm d$. The dots are small, with $\beta=0.01 \mathrm{~nm}^{-2}$, or larger with $\beta=0.003 \mathrm{~nm}^{-2}$ in Eq. (11). The depth of the dots or wells is $V_{0}=-10 \mathrm{meV}$, and we assume GaAs parameters yielding the effective Bohr radius $a_{0}=9.79 \mathrm{~nm}$, and the Rydberg $R y=5.92 \mathrm{meV}$. Clearly, we can expect the transport to vary strongly with the width of the wire since in the case of the broad wire several subbands or modes can be coupled by the scattering potential. In the narrow wire the characteristic length $a_{w}=\sqrt{\hbar /\left(m \Omega_{0}\right)}=$ $13.8 \mathrm{~nm}$, in the broad one $a_{w}=33.7 \mathrm{~nm}$.

\section{A. Narrow wire}

The conductance of the narrow wire is shown in Fig. 11 for both types of dots as function of the parameter $X=E / E_{0}+1 / 2$, whose integral part numbers the propagating subbands participating in the transport at a particular energy $E$. The figure indicates that the main role of the dots in the narrow wire is to define a semitransparent cavity in between them. In the case of the small dots this cavity has a well defined length and well known geometrical resonances are seen in the conductance as the separation, $d$, of the dots is increased. When the dots are larger (lower panel) the higher order resonances vanish and one or two dips occur in the conductance, characteristic of an attractive scattering potential. For the smallest separation of the dots, when they partially overlap we see two dips. In Fig. 2 we present the probability density for three relevant cases in order to better understand the microscopic processes in the system. In Fig. 2(a) we see the probability density for the large dots corresponding to the lowest resonance in the lower panel of Fig. 1] when $d=8 a_{0}$. As expected the main probability density is located in the cavity between the dots. Interestingly, due to the finite size of the dots we also see a small density at the location of each dot. Here the electrons enter the system in the lowest mode $n=0$ and exit the system in the same mode, this together with the total transmission leads to a constant probability density in the wire away from the scattering potential. In Fig. 2(b) and (c) we see the probability density at the dips for the large dots as $d=2 a_{0}$. The two states causing the dips are the symmetric and the anti-symmetric quasi-bound state the latter one occurring at a bit higher energy as expected. The structure of the density in either case reminds us that due to the high symmetry of the dot potential, Eq. (1), the evanescent state causing the reflection of the $n=0$ mode is in the third subband with $n=2$ and is thus broader than the resonant state in Fig. 2(a). Due to the high probability density of the electrons in the evanescent state we do barely see the incoming and the reflected density on the left side of the
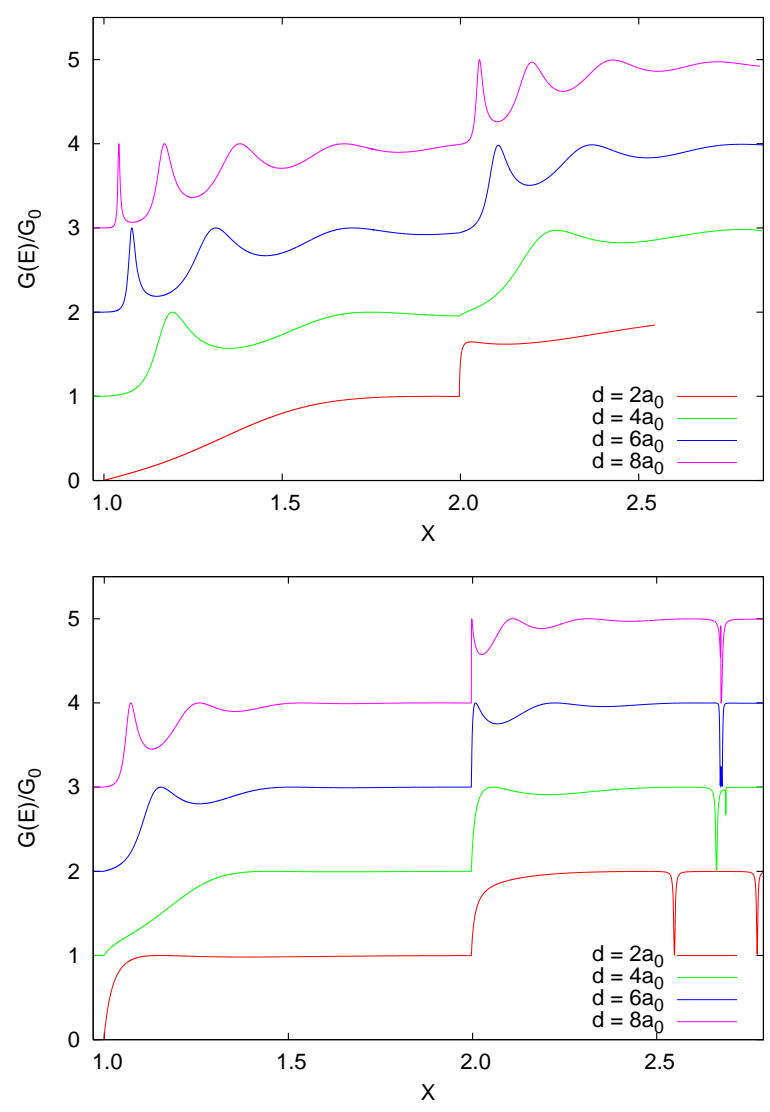

FIG. 1: The conductance in units of $G_{0}=2 e^{2} / h$ of a narrow parabolic wire with embedded small dots $\beta a_{w}^{2}=1.90$ (upper panel), and large dots $\beta a_{w}^{2}=0.571$ (lower panel) for different displacement $z= \pm d$ from the center of the wire $z=0$ as function of $X=E / E_{0}+1 / 2 . E_{0}=\hbar \Omega_{0}=6.0 \mathrm{meV}, V_{0}=-10$ $\mathrm{meV}, a_{w}=13.8 \mathrm{~nm}$, and for GaAs $a_{0}=9.79 \mathrm{~nm}$.

scattering potential on the chosen color scale.

\section{B. Broad wire}

In order to prepare the analyses of the conduction of a broad quantum wire $\left(E_{0}=1 \mathrm{meV}\right)$ with two embedded dots we first show in the upper panel of Fig. 3] the conduction in the broad wire with only one quantum dot embedded, large or small. The sharp dip seen at the end of the second conduction step in a narrow wire is turned into a broad minimum in the broad wire due to the stronger coupling between the subbands caused by the dot in the broad wire.

In the lower panel of Fig. 3 we display the conductance of the broad wire with the two embedded dots at $d=8 a_{0}$. The presence of the two well separated dots adds considerably to the fine structure of the conductance compared to the results for one dot. Moreover, this structure involves the coupling of many subbands and thus requires the inclusion of approximately 16 of them in the numerical calculation in order to reach well converged results. 

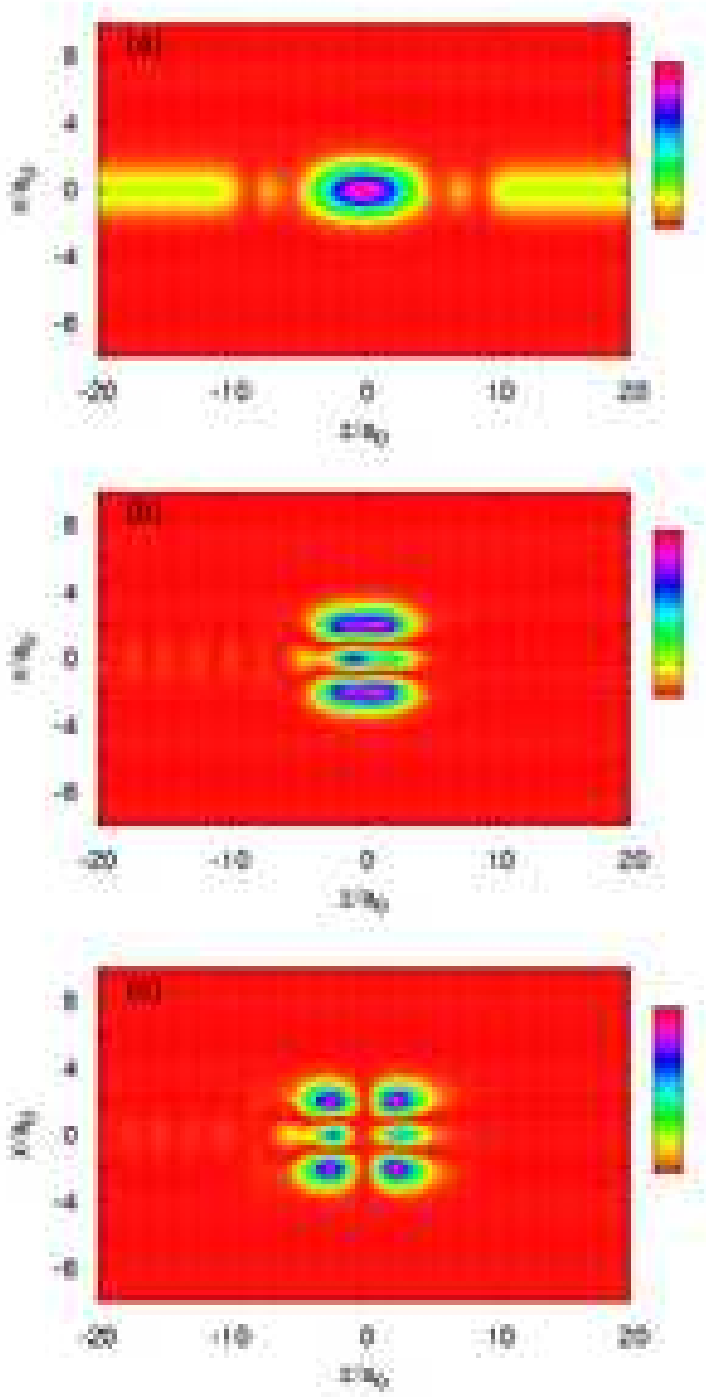

FIG. 2: The probability density of the scattering state $\psi_{E}(x, y)$ in the narrow quantum wire in the presence of two embedded large dots. The incident energy of the $n=0$ mode corresponds to $X=1.08$ and $d=8 a_{0}$ (a), $X=2.58$ and $d=2 a_{0}$ (b), and $X=2.80$ and $d=2 a_{0}$ (c), corresponding to the peak and the two dips, respectively, in the conductance in the lower panel of Fig. 1 $E_{0}=\hbar \Omega_{0}=6.0 \mathrm{meV}, V_{0}=-10$ $\mathrm{meV}, a_{w}=13.8 \mathrm{~nm}, \beta a_{w}^{2}=0.571$.

First, we start with the electron probability density shown in Fig. 4 to gain insight into the multitude of processes taking place in the case of the small dots. Similarly, as in the case of the narrow wire we have a transmission resonance at $X=1.284$ caused by a state located in the "cavity" between the dots shown in Fig. 4(a). We also see small probability maxima at the dots themselves. The second transmission resonance at $X=2.07$ is caused by the next mode in the inter-dot cavity and is shown in
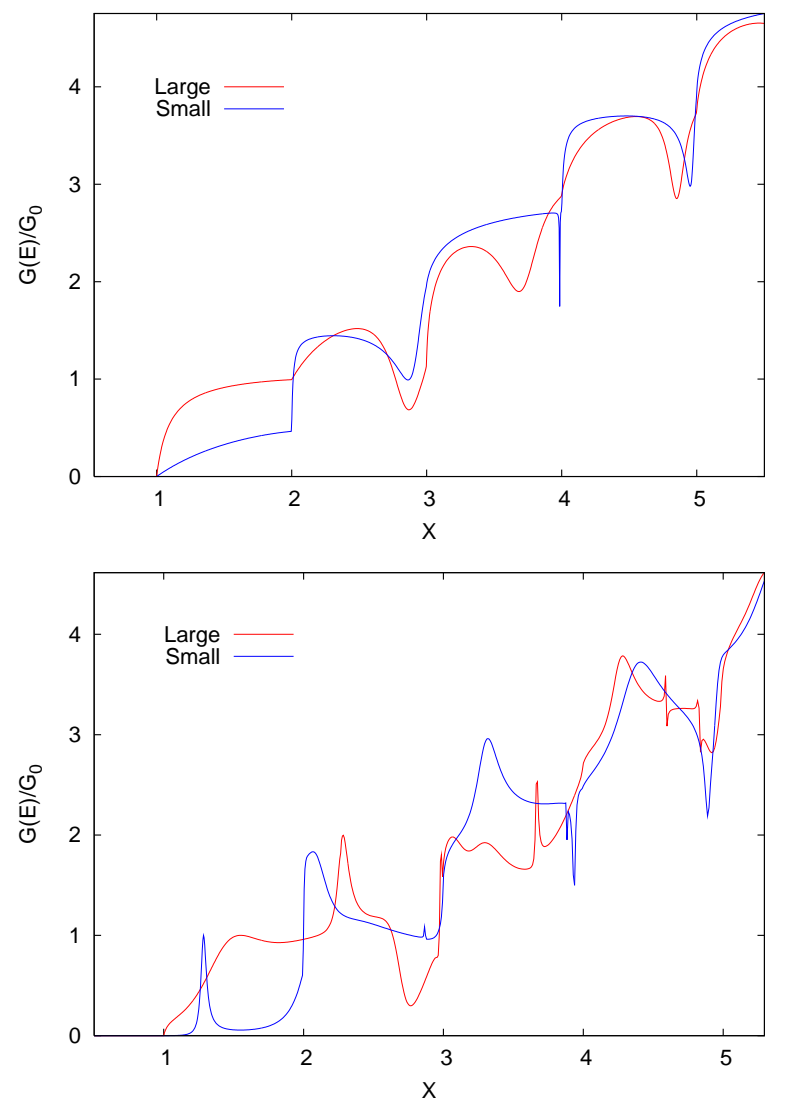

FIG. 3: The conductance in units of $G_{0}=2 e^{2} / h$ of a broad wire with one embedded $\operatorname{dot}$ at the center $z=0$, (upper panel), and two dots at $z= \pm 8 a_{0}$, (lower panel) as function of $X=E / E_{0}+1 / 2 . E_{0}=\hbar \Omega_{0}=1.0 \mathrm{meV}, V_{0}=-10 \mathrm{meV}$, $a_{w}=33.7 \mathrm{~nm}$, and for GaAs $a_{0}=9.79 \mathrm{~nm}$. A small dot is characterized by $\beta a_{w}^{2}=11.37$ and a large one by $\beta a_{w}^{2}=3.41$.

Fig. I4(c), but it is also interesting to check the probability density at a bit lower energy $X=1.98$, see 4l(b). Here there is considerable reflection and as the Figure shows this can be interpreted as the electrons being reflected by the second dot, the first dot simply loses its effects in a minimum in the interference between the incoming and reflected wave. Thus, by increasing the energy to $X=2.76$ we observe a reflection of the first dot in 4 (d). The valley structure in $G$ around $X=2.76$ corresponds to the electrons making inter-subband transitions from $n=0$ to the subband threshold of the third subband $(n=2)$ forming a quasi-bound state. The wide valley structure implies a short life time of such a state.

At $X=2.86$ we see a narrow resonance in the conduction. The corresponding probability density in Fig. 5 has the typical structure of an evanescent state in the third subband for the incoming $n=0$ state and a normal conducting mode for the $n=1$ instate. The evanescent state clearly belongs mainly to the first dot, but is also fairly extended into the inter-dot cavity.

To finish the observation of the case of the small dots we examine the electronic probability density for the two 

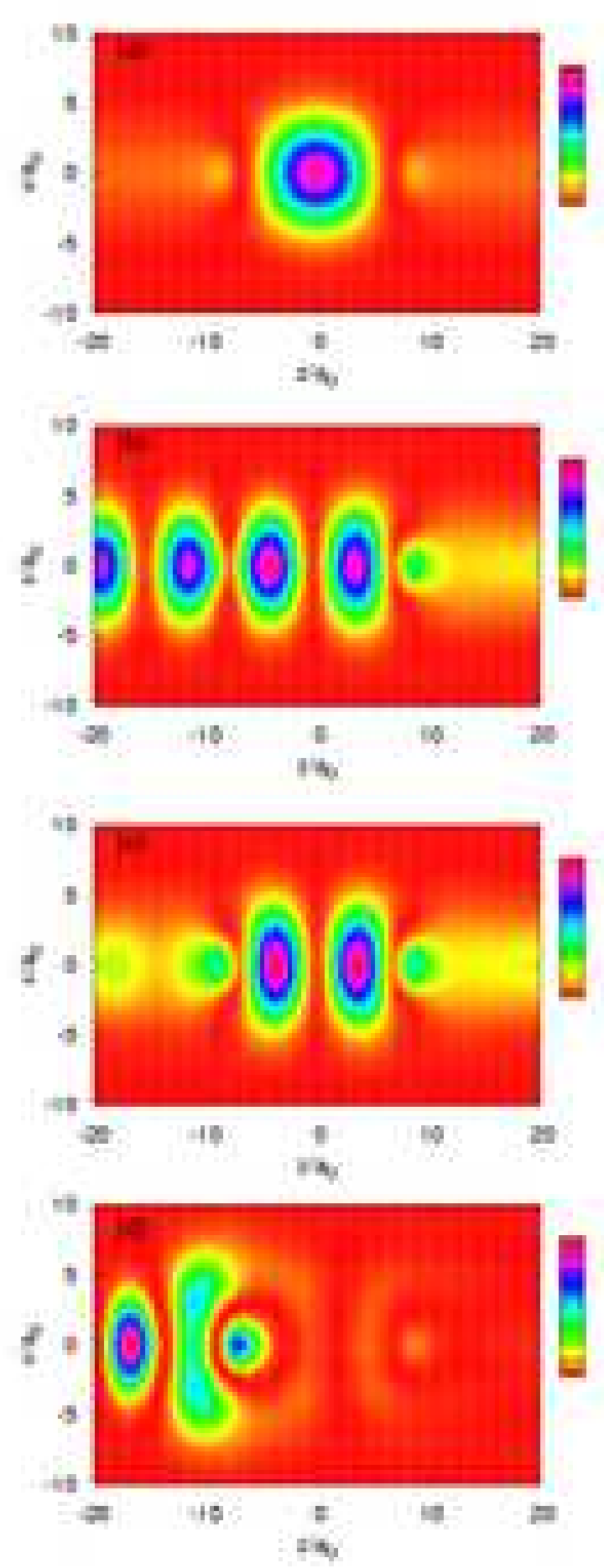

FIG. 4: The probability density of the scattering state $\psi_{E}(x, y)$ in the broad quantum wire in the presence of two small embedded dots centered at $z= \pm 8 a_{0}$. The incident energy and modes correspond to: (a) $X=1.284$ and $n=0$, (b) $X=1.98$ and $n=0$, (c) $X=2.07$ and $n=0$, and (d) $X=2.76$ and $n=0 . E_{0}=\hbar \Omega_{0}=1.0 \mathrm{meV}, V_{0}=-10 \mathrm{meV}$, $a_{w}=33.7 \mathrm{~nm}$, and $\beta a_{w}^{2}=11.37$.
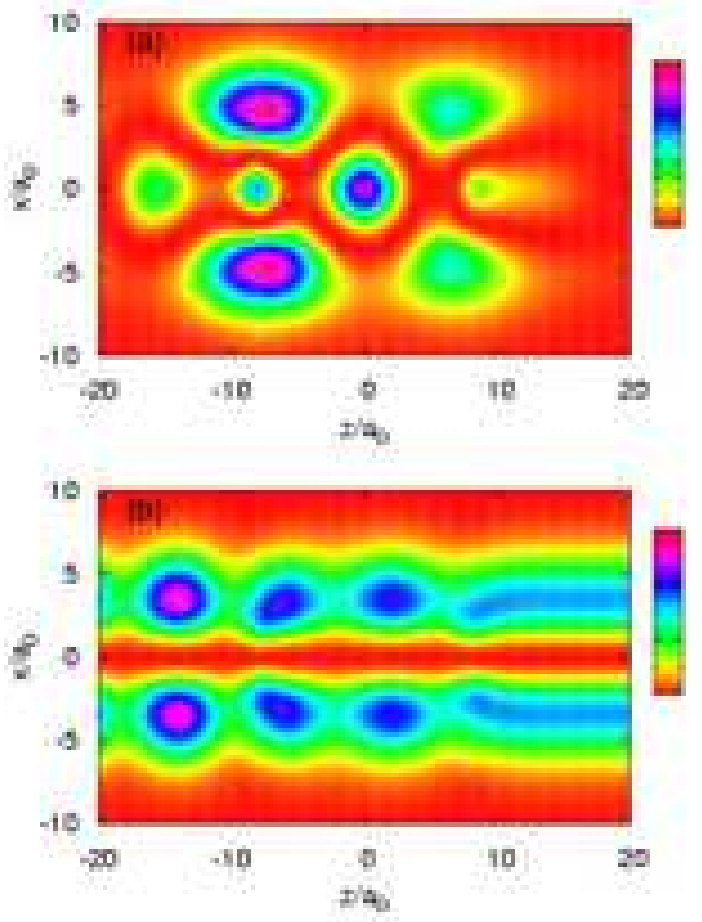

FIG. 5: The probability density of the scattering state $\psi_{E}(x, y)$ in the broad quantum wire in the presence of two small embedded dots centered at $z= \pm 8 a_{0}$. The incident energy and modes correspond to: (a) $X=2.86$ and $n=0$, (b) $X=2.86$ and $n=1 . E_{0}=\hbar \Omega_{0}=1.0 \mathrm{meV}, V_{0}=-10 \mathrm{meV}$, $a_{w}=33.7 \mathrm{~nm}$, and $\beta a_{w}^{2}=11.37$.

narrow dips found at $X=3.88$, and 3.94. These are presented in Fig. [6 for the two lowest incoming modes $n=0$ and 1 . Here the first and the third mode conduct partially, as can be confirmed for the $n=0$ mode in Fig. 6) (a) and (c). The two $n=0$ modes are almost identical, but the second incoming mode $n=1$ is reflected due to an interaction with an evanescent mode in the fourth $n=3$ subband. The evanescent modes in Fig. 6(b) and (d) show the symmetry of the fourth band and are the distinct two lowest symmetric and antisymmetric quasibound states of the dot system.

The system with larger dots allows for a richer mixture of intra and inter-dot processes or states. The lowest transmission resonance is now a broad feature with a corresponding electronic probability density that is a clear superposition of a state in the inter-dot cavity and states located at each dot, see Fig. [7 (a). The next transmission resonance at $X=2.28$ is narrow and for the incoming $n=0$ mode is composed of a state with a high proportion of a $p_{z}$-type atomic orbital or higher on each dot as the probability in Fig. I(b) shows. The $n=1$ in-mode on the other hand is basically a $p_{y}$-type state in the interdot cavity and to lesser degree $p_{y}$-states at each dot, see Fig. 7 (c). In the conductance minimum at $X=2.77$ we 

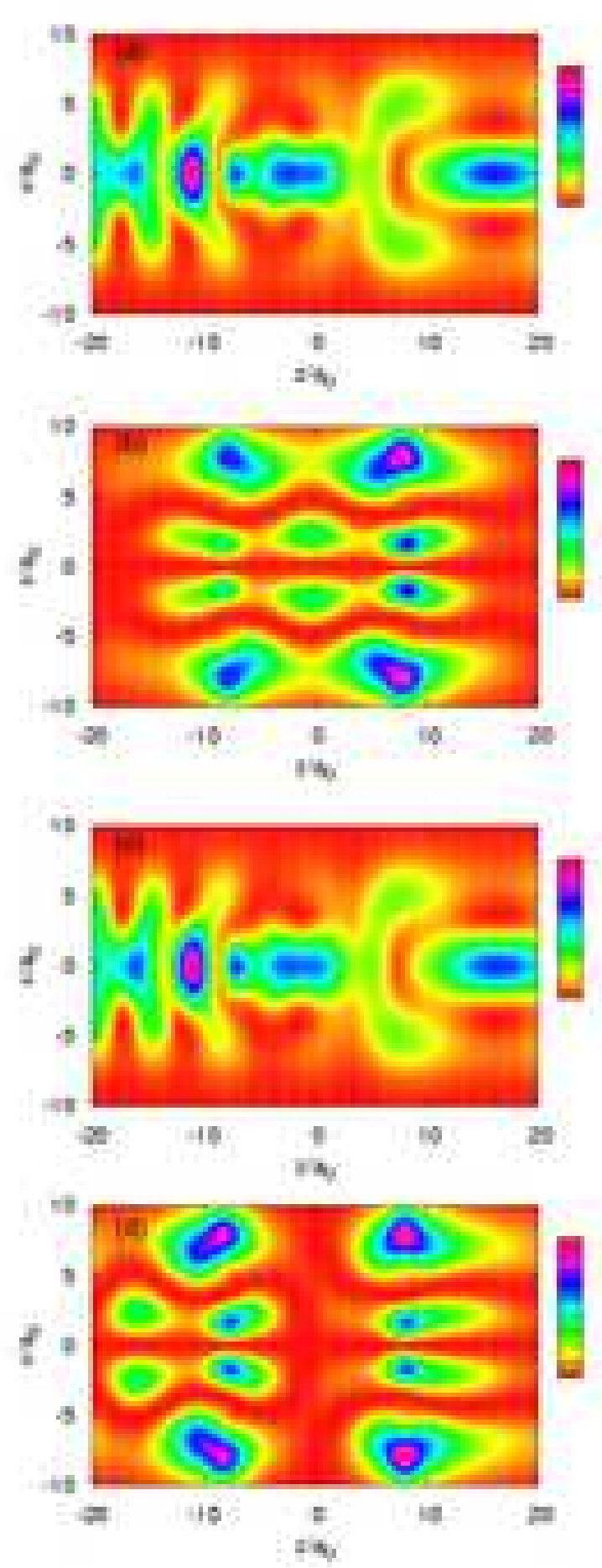

FIG. 6: The probability density of the scattering state $\psi_{E}(x, y)$ in the broad quantum wire in the presence of two small embedded dots centered at $z= \pm 8 a_{0}$. The incident energy and modes correspond to: (a) $X=3.88$ and $n=0$, (b) $X=3.88$ and $n=1$, (c) $X=3.94$ and $n=0$, and (d) $X=3.94$ and $n=1 . E_{0}=\hbar \Omega_{0}=1.0 \mathrm{meV}, V_{0}=-10 \mathrm{meV}$, $a_{w}=33.7 \mathrm{~nm}$, and $\beta a_{w}^{2}=11.37$.
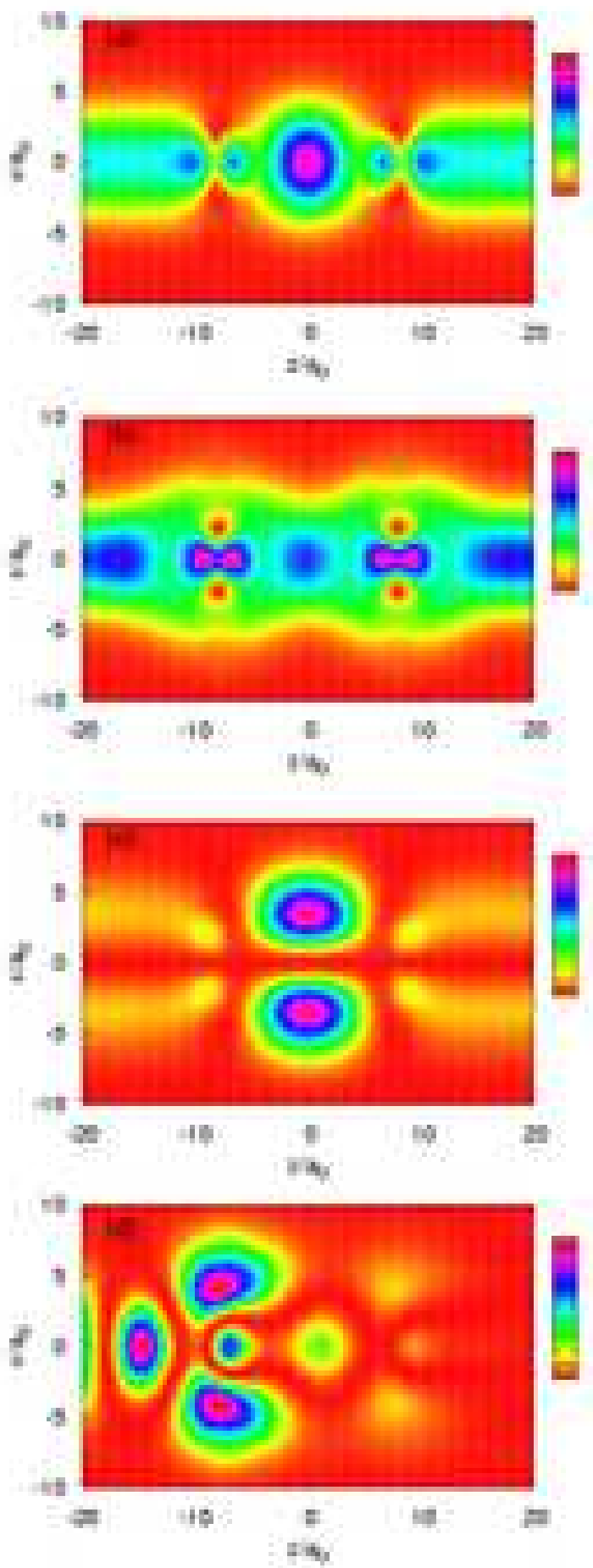

FIG. 7: The probability density of the scattering state $\psi_{E}(x, y)$ in the broad quantum wire in the presence of two large embedded dots centered at $z= \pm 8 a_{0}$. The incident energy and modes correspond to: (a) $X=1.55$ and $n=0$, (b) $X=2.28$ and $n=0$, (c) $X=2.28$ and $n=1$, (d) $X=2.77$ and $n=0 E_{0}=\hbar \Omega_{0}=1.0 \mathrm{meV}, V_{0}=-10 \mathrm{meV}, a_{w}=33.7$ $\mathrm{nm}$, and $\beta a_{w}^{2}=3.41$. 
observe the familiar evanescent mode (Fig. $\mathbf{7}(\mathrm{d})$ ) in the third subband reflecting a large portion of the incoming wave.

At $X=3.67$ the conductance displays a narrow resonance that is caused by an evanescent state in the fourth subband interacting with the second incoming mode as can be verified by the probability density shown in Fig. 8b). Here is also curious to note that the scattering potential mixes up the first and the third incoming modes; Fig. 8(a) shows the incoming $n=0$ state leaving the scattering region in the $n=2$ mode, and the opposite process is seen happening in Fig. 8 (c). In the first case there is a considerable probability for the electron in the first dot, and in the second case this is reversed. Here, one should remember that even though we talk about large dots in this case the effective size of the dots is comparable to the width of the incoming state and thus the third mode is quite broader than the dot, and the broadness of the wire leads to large matrix elements between the the first and the third mode of the wire. This mixing of incoming states, a character of nonadiabatic transport, or crosstalk between the channels or modes, is not limited to the peak at $X=3.67$. At the broader peak at $X=4.3$ the same also happens for the odd modes $n=2$ and $n=4$.

As expected, for higher energy the conductance of the broad wire becomes similar for the small and the larger dots except for the Fano resonances that represent quasibound states present in the energy continuum that depend on the shape of the pertinent dots.

\section{SUMMARY}

We have explored the transport through a quantum wire with two embedded quantum dots that are not broader then the wire. We find in the transport an interplay between intra- and inter-dot scattering processes. In particular, we find for the larger dots in a broad wire drastic expamles of nonadiabatic transport 13]. Bryant explores the transport through a hard-wall quantum wire with a quantum dot defined by a tapered center and separated from the wire region by two rectangular barriers [13]. He finds the mode-mixing effects to be more important when the transmission occurs by resonant tunneling and when the tapering is not quite smooth. Here we observe strong mode-mixing in ballistic transport when the energy subbands of the wire are closely spaced on the scale of the depth of the quantum dots and the size of the dots results in large matrix elements between the various transverse modes. Here is important that the symmetry of the centered dots establishes selection rules for the matrix elements that are thus of very variable magnitude. Also, even though one quantum dot of Gaussian shape can be considered smooth, then it is more difficult to measure the smoothness of the system of two smooth dots very well separated.

The methods employed to calculate the conductance have been applied to wires with parabolic (here) and hard
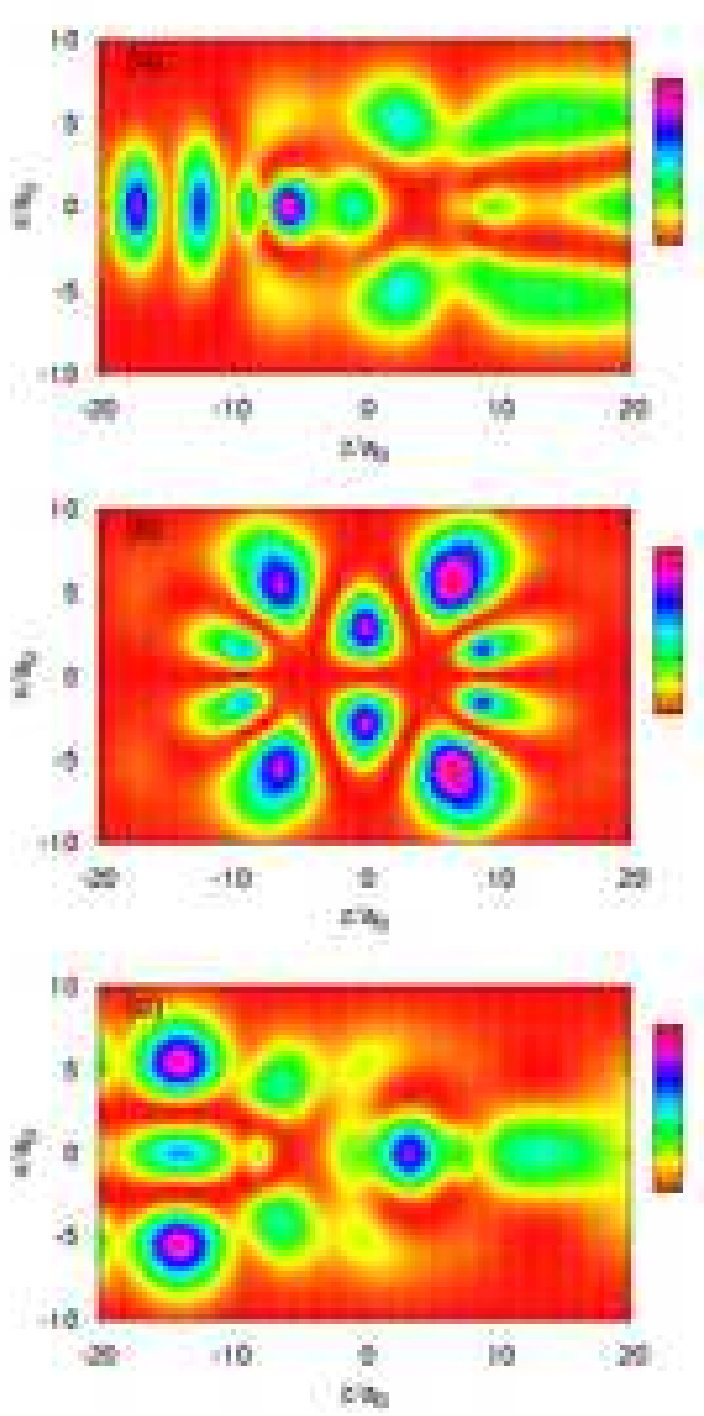

FIG. 8: The probability density of the scattering state $\psi_{E}(x, y)$ in the broad quantum wire in the presence of two large embedded dots centered at $z= \pm 8 a_{0}$. The incident energy and modes correspond to: (a) $X=3.67$ and $n=0$, (b) $X=3.67$ and $n=1$, and (c) $X=3.67$ and $n=2$ $E_{0}=\hbar \Omega_{0}=1.0 \mathrm{meV}, V_{0}=-10 \mathrm{meV}, a_{w}=33.7 \mathrm{~nm}$, and $\beta a_{w}^{2}=3.41$.

wall confinement [19] in the transverse direction and are applicable to general scattering potentials as long as care is taken in selecting a sufficient number of input modes.

\section{Acknowledgments}

The research was partly funded by the Research and Instruments Funds of the Icelandic State, and the Research Fund of the University of Iceland. C.S.T. acknowledges computational facilities supported by the National 
Center for High-performance Computing in Taiwan.

[1] R. H. Blick, R. J. Haug, J. Weis, D. Pfannkuche, K. v. Klitzing, and K. Eberl, Phys. Rev. B 53, 7899 (1996).

[2] R. H. Blick, D. Pfannkuche, R. J. Haug, K. Klitzing, and K. Eberl, Phys. Rev. Lett. 80, 4032 (1998).

[3] H. Jeong, A. M. Chang, and M. R. Melloch, Science 293, 2221 (2001).

[4] A. W. Holleitner, R. H. Blick, A. K. Hüttel, K. Eberl, and J. P. Kotthaus, Science 297, 70 (2002).

[5] M. Rontani, S. Amaha, K. Muraki, F. Manghi, E. Molinari, S. Tarucha, and D. G. Austing, Phys. Rev. B 69, 085327 (2004).

[6] G. Klimeck, G. Chen, and S. Datta, Phys. Rev. B 50, 2316 (1994).

[7] C. Niu, L.-jun Liu, and T.-han Lin, Phys. Rev. B 51, 5130 (1994).

[8] C. A. Stafford and S. D. Sarma, Phys. Rev. Lett. 72, 3590 (1994).

[9] A. Aharony, O. Entin-Wohlmann, Y. Imry, and Y. Levinson, Phys. Rev. B 62, 13561 (2000).

[10] P. A. Orellana, G. A. Lara, and E. V. Anda, Phys. Rev. B 65, 155317 (2002).
[11] M. L. L. de Guevara, F. Claro, and P. A. Orellana, Phys. Rev. B 67, 195335 (2004).

[12] V. Moldoveanu, A. Aldea, and B. Tanatar, Phys. Rev. B 70, 085303 (2004).

[13] G. W. Bryant, Phys. Rev. B 44, 12837 (1991).

[14] C. S. Kim, A. M. Satanin, Y. S. Joe, and R. M. Cosby, Phys. Rev. B 60, 10962 (1999).

[15] S. A. Gurvitz and Y. B. Levinson, Phys. Rev. B 47, 10578 (1993).

[16] J. U. Nöckel and A. D. Stone, Phys. Rev. B 50, 17415 (1994).

[17] P. F. Bagwell, Phys. Rev. B 41, 10354 (1990).

[18] G. Cattapan and E. Maglione, Am. J. Phys. 71, 903 (2003).

[19] J. H. Bardarson, I. Magnusdottir, G. Gudmundsdottir, C.-S. Tang, A. Manolescu, and V. Gudmundsson, (cond-mat/0408435) (2004).

[20] M. Buttiker, Y. Imry, R. Landauer, and S. Pinhas, Phys. Rev. B 31, 6207 (1985).

[21] D. S. Fisher and P. A. Lee, Phys. Rev. B 23, 6851 (1981). 